\title{
eJRIEPS
}

Ejournal de la recherche sur l'intervention en éducation physique et sport

9 | 2006

Varia

\section{Enseigner le badminton à une classe de primo arrivants : incidence du contexte ZEP (zone d'éducation prioritaire) sur la pratique d'intervention}

Claire Debars et Chantal Amade-Escot

\section{(2) OpenEdition Journals \\ Édition électronique \\ URL : https://journals.openedition.org/ejrieps/7359 \\ DOI : 10.4000/ejrieps.7359 \\ ISSN : 2105-0821 \\ Éditeur \\ ELLIADD}

Édition imprimée

Pagination : 35-43

\section{Référence électronique}

Claire Debars et Chantal Amade-Escot, «Enseigner le badminton à une classe de primo arrivants incidence du contexte ZEP (zone d'éducation prioritaire) sur la pratique d'intervention », eJRIEPS [En ligne], 9 | 2006, mis en ligne le 01 janvier 2006, consulté le 29 octobre 2021. URL : http:// journals.openedition.org/ejrieps/7359; DOI : https://doi.org/10.4000/ejrieps.7359

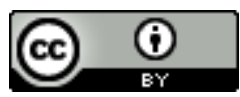

La revue eJRIEPS est mise à disposition selon les termes de la Creative Commons Attribution 4.0 International License. 
Enseigner le badminton à une classe de primo arrivants : incidence du contexte ZEP (zone d'éducation prioritaire) sur la pratique d'intervention.

\author{
Claire Debars \& Chantal Amade-Escot \\ ID2-LEMME, Université Paul Sabatier, Toulouse III
}

\title{
Résumé
}

Les principaux résultats de cette recherche exploratoire sur l'action didactique d'un professeur d'éducation physique et sportive (EPS) en zone d'éducation prioritaire (ZEP) ont permis de mettre à jour les contraintes qui pèsent sur la pratique enseignante dans ce qu'il est convenu d'appeler un "milieu difficile". Ils montrent un souci de contrôle des élèves entraînant un éclatement du contenu initialement visé par la mise en place en cours des séances d'une succession de micro tâches ; le développement de stratégies très individualisées de régulation de l'activité des élèves; et enfin, un grand nombre d'interactions verbales. Ces constats pointent des difficultés à laisser une certaine autonomie aux élèves, cependant, l'observation met en évidence que le rappel systématique des consignes de réalisation et d'organisation de l'espace, contribue, dès lors que le milieu didactique est suffisamment ouvert, à créer des opportunités d'apprentissage.

Dans le cadre du symposium sur «l'intervention dans les milieux défavorisés » notre communication a pour but d'analyser l'activité d'un professeur enseignant le badminton dans un collège situé en zone d'éducation prioritaire (ZEP). Nous souhaitons comprendre quelle est l'incidence du contexte ZEP sur son action didactique, quelles sont les contraintes qui pèsent sur elle et les ressources qui sont susceptibles d'être mobilisées au fil des interactions avec les élèves.

\section{Problématique}

Des précédentes études en sociologie de l'éducation, en didactique des mathématiques et en didactique de l'éducation physique et sportive (EPS), ont montré la difficulté que rencontraient les enseignants de ZEP pour maintenir les enjeux de savoir initialement envisagés et/ou préconisés par les programmes 
(Bautier et Rochex, 1997 ; Butlen, Peltier-Barbier et Pézart, 2002 ; Badreau et Dhellemmes, 1998 ; Peltier-Barbier, 2004 ; Van Zanten, 2001). Ces différents auteurs ont mis en évidence des processus de réduction des exigences relatives aux contenus enseignés au profit d'une intervention mettant en avant des objectifs de socialisation ou d'éducation citoyenne. A partir d'une approche didactique, nous reprenons cette question et nous tentons de comprendre les processus qui sont à l'œuvre en EPS et notamment ceux qui font que les savoirs enseignés en ZEP tendent à être réduits par rapport à ceux enseignés en établissement tout venant (Poggi-Combaz, 2002). A partir d'une étude de cas (un cycle de badminton) nous décrivons le fonctionnement d'un système didactique ${ }^{1}$ en ZEP, dans un but d'identifier, d'une part, les contraintes qui pèsent sur l'action didactique du professeur et d'autre part, les espaces potentiels de développement des contenus qui restent possibles. L'objectif de la recherche vise à mieux comprendre comment les enseignants régulent les apprentissages face aux contraintes de ce qu'il est convenu d'appeler « un milieu difficile ».

\section{Cadre théorique et question de recherche.}

A partir des résultats de recherches précédemment citées, nous avons identifié un certain nombre d'éléments susceptibles d'avoir une influence sur les pratiques d'intervention en EPS. Van Zanten (2001) décrit, lors d'une étude ethnographique, les contraintes fonctionnelles et éthiques qui sont à l'origine de compromis entre enseignants et élèves pour faire du collège un lieu de vie social autour de normes relationnelles et comportementales qui prennent le pas sur la réalisation du programme officiel. Bautier et Rochex (1997) constatent que les effets de la politique ZEP ont davantage porté sur des actions visant, d'une part, à une meilleure socialisation des élèves et, d'autre part, à la prévention des violences et des incivilités au détriment de l'appropriation des contenus de savoir. Une recherche récente (Peltier, Butlen et Pézard, 2002), en didactique des mathématiques, confirme que la socialisation des élèves à l'école primaire est privilégiée par rapport aux contenus enseignés. Leurs travaux signalent une réduction des exigences relatives

\footnotetext{
${ }^{1}$ Le système didactique est défini comme « un système de relations entre le jeu de l'enseignant et le jeu de l'élève à propos du savoir à enseigner et à apprendre, sous couvert d'institutions dotées d'intention d'enseignement » (Schubauer-Leoni, 1996).
} 
aux contenus du savoir mathématique en ZEP et en réseau d'éducation prioritaire (REP). En EPS, Badreau et Dhellemmes (1998), lors d'une recherche visant à évaluer les effets de séquences tirées d'ingénieries didactiques sur l'enseignement en milieu difficile, démontrent qu'il est toutefois possible de maintenir des contenus d'enseignement dès lors que les situations proposées sont pertinentes. Cependant, ces auteurs révèlent la nécessité d'instaurer un climat favorable et de consacrer davantage de temps aux situations. Pour conclure, la revue de littérature pointe une réelle difficulté à maintenir les exigences sur les contenus à enseigner en ZEP. Qu'en est-il en éducation physique dans le cadre d'un enseignement ordinaire non préparé en amont par une action des chercheurs ? Que deviennent les contenus d'enseignement au fil des interactions didactiques ? La réduction des savoirs enseignés est-elle inévitable ? Quelles sont les marges de manœuvre laissées à l'enseignant?

\section{Méthodologie}

Pour répondre à ces questions et caractériser l'activité enseignante en ZEP nous avons observé deux classes de $4^{\text {ème }}$ dans deux collèges différents (un collège ZEP et un collège tout venant) afin de pouvoir comparer le fonctionnement des systèmes didactiques.

Les élèves du collège classé ZEP, au sein duquel nous avons mené nos observations, sont issus de quartiers défavorisés. Les parents sont en majorité des immigrés en situation précaire. La classe observée est une classe d'accueil, composée d'élèves primo arrivants de plusieurs nationalités. Certains élèves de cette classe n'étaient pas scolarisés dans leur pays d'origine. Ils ont de 11 à 15 ans. D'un point de vue scolaire, ils ont des difficultés de compréhension et d'expression en français. L'enseignant trouve cependant que cette classe est moins difficile à gérer d'un point de vue disciplinaire que d'autres classes et c'est la raison pour laquelle il a bien voulu nous accepter en tant qu'observateur. Notons que, dans le cadre de cet article, nous nous limiterons à décrire le travail de l'enseignant en ZEP.

Pour recueillir les données nécessaires à notre étude nous nous sommes inspirées de la méthode d'observation développée par Schubauer-Leoni et Leutenegger (2002) et remaniée pour l'EPS par Amade-Escot (2003). Elle combine des données d'entretiens (ante-cycle, ante-séances et post-séances) permettant d'accéder au point de vue du professeur et des données d'observation par enregistrement vidéo et 
audio. Les interactions verbales et comportementales entre le professeur et les élèves au cours des situations d'apprentissage de trois séances consécutives, situées au milieu d'un cycle de six séances, ont fait l'objet de l'analyse. La triangulation des données (Miles et Huberman, 1991) est à l'origine des résultats que nous présentons. L'action du professeur a été analysée en mettant en relation le type de situations proposées aux élèves, le contenu des interactions verbales de l'enseignant et des élèves et, plus particulièrement, les régulations effectuées par le premier en relation avec les comportements des seconds lors des tâches d'apprentissage dont nous avons fait l'analyse a priori (Mercier et Salin, 1988). Le croisement de ces différentes données nous a permis de décrire l'évolution de ce système didactique au fil des transformations des milieux didactiques.

Résultats et interprétations

Les principaux résultats mettent en évidence chez le professeur observé un souci de contrôle des élèves, des régulations très individuelles et un grand nombre d'interactions verbales. Deux exemples seront successivement présentés.

Un souci de contrôle des élèves entraînant un éclatement des contenus visés en micro tâches D'une manière récurrente, les observations mettent en évidence que l'enseignant répartit rapidement les élèves sur les terrains de badminton, donne les consignes initiales et passe de terrain en terrain en s'adressant à chacun des élèves individuellement. II déploie une très grande activité de régulation auprès des élèves lorsque ceux-ci sont en train de réaliser le travail demandé. II formule des remarques qui portent essentiellement sur le rappel des consignes et sur l'organisation de l'espace. Au départ des situations, les dispositifs initiaux, plutôt pertinents par rapport à la logique de l'activité du badminton, proposent des tâches ouvertes avec plusieurs variables comme le travail du service court et long ou celui sur les trajectoires variées dans la largeur et dans la longueur. Mais, le souci de maintenir les élèves en activité amène l'enseignant à réduire très rapidement les tâches. La gestion de la séance révèle une stratégie d'enseignement visant à contrôler l'activité motrice des élèves, notamment, en réduisant les alternatives décisionnelles, comme l'exemple ci-après en rend compte

Alors que l'enseignant a choisi de faire travailler les élèves sur la variable court et long (entretien ante séance), il propose la situation suivante. La tâche proposée aux élèves consiste à produire une trajectoire de service devant atteindre le carré dessiné sur le terrain. Le réceptionneur « $R$ » doit se placer entre les deux lignes du 
fond, parallèles au filet. L'analyse a priori pointe ici que l'alternative prévue initialement (entretien ante séance) se trouve immédiatement réduite à la production d'un service court, dans le carré dessiné au sol.

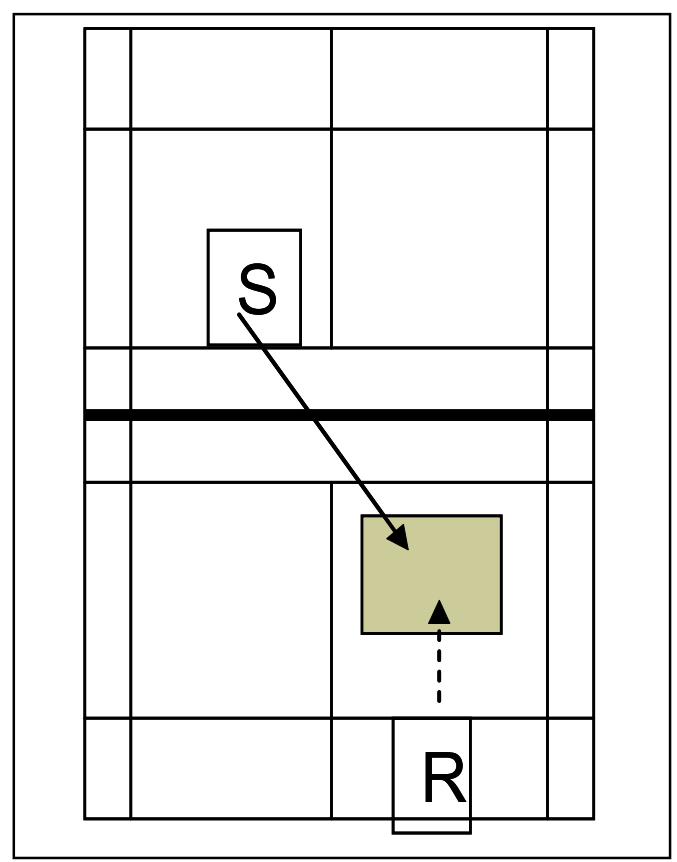

Figure 1. Descriptif de la tâche prescrite aux élèves

Les élèves se mettent par deux avec un volant sur un terrain. Le joueur " $S$ » sert. La consigne est d'enchaîner plusieurs services courts : "le service doit être efficace immédiatement ». Très rapidement, nous observons que les élèves servent pour mettre en jeu et non pour mettre l'adversaire en difficulté. Or, l'enjeu tactique consiste à envoyer le volant là où n'est pas l'adversaire. Aucune indication n'est donnée au joueur réceptionneur « $R$ ». L'analyse a priori laisse penser que l'élève au service peut, selon le placement du réceptionneur, alterner un service court ou long. Cependant les rétroactions du milieu (le carré dessiné au sol et la consigne d'efficacité immédiate) réduisent d'emblée la tâche à un micro exercice de pointage (envoyer le volant sur une cible horizontale délimitée).

L'analyse de l'évolution de la situation met en évidence une réduction du savoir par rapport à l'intention didactique initiale. En effet, il faut viser un espace précis. Le milieu didactique est construit par le biais d'un aménagement : la zone près du filet. Cet aménagement crée une forte rétroaction du milieu. Mais les élèves ne construisent pas réellement le sens tactique du service. Nous avons d'ailleurs 
observé que le réceptionneur, « $R$ » se place volontairement dans la zone où tombe le volant, (et non entre les deux lignes parallèles du fond du court) au fur et à mesure du déroulement de la situation. Progressivement la tâche initialement pensée pour introduire l'alternative servir court ou servir long éclate en plusieurs micro tâches centrés sur l'effectuation d'un geste : servir court, puis échanger en duo avec un partenaire placé au point de chute du volant.

\section{Des régulations très individuelles}

Pour exemplifier ce que nous considérons être une contrainte liée au contexte ZEP, nous commentons une situation de jonglage proposée pendant la $4^{\text {ème }}$ séance $\mathrm{du}$ cycle. L'enseignant, selon ses propres dires, cherche à faire repérer aux élèves « la distance entre l'épaule et le tamis » (entretien ante séance). Les consignes données aux élèves sont d'effectuer des «jonglages sans envoyer le volant trop haut et en gardant le bras tendu ». L'extrait ci-après illustre le type de régulations émises par l'enseignant :

Vous vous repartissez sur les terrains ! L'enseignant s'adresse à un élève : Rouan, bras tendu ! Voilà ! II s'adresse à la classe : il y a de la place sur tout le gymnase. Vous êtes trop serrés. II revient sur Rouan : Rouan écarte-toi ! II s'approche de Manel : Manel écarte toi ! A un autre élève : Senay, s'il te plait ! Il y a de la place ! De nouveau, il insiste sur les consignes et répète trois fois : Bras tendu ! Puis, il s'obstine sur l'organisation de l'espace : Il y a de la place là-bas sur les terrains !

A travers cet extrait, nous constatons que l'activité principale de l'enseignant consiste à gérer l'espace de travail. Pour éviter que les élèves soient inactifs, il leur demande de s'installer, puis les houspille sans cesse pour qu'ils occupent à bon escient l'espace de travail. En même temps, il intervient sans cesse pour réguler l'activité motrice des élèves. II les contrôle en permanence en donnant des injonctions sur l'action à effectuer et en précisant comment l'effectuer : « Vite tends ton bras / c'est ça / tends ton bras Mina ». On retrouve ici une individualisation exacerbée de l'activité régulatrice assez proche de celle mise en évidence par Peltier-Barbier (2004) en mathématiques. Pour conclure, nous avons interprété cette stratégie comme un moyen de maintenir les élèves en activité tout en les contrôlant pour éviter des risques de déviance.

Le grand nombre d'interactions verbales 
Le troisième constat effectué souligne la densité du flux des interventions verbales de l'enseignant. Nous utilisons pour le mettre en évidence un élément de comparaison avec le cycle observé en collège tout venant. Le nombre d'interactions est cinq fois plus élevé en ZEP (447 tours de paroles pour la classe de primo arrivants et 87 pour l'autre classe). Cela laisse peu d'autonomie aux élèves. Trois hypothèses peuvent être avancées. La première hypothèse reprend le constat précédent d'un souci de contrôle quasi permanent des élèves et de leur activité. La deuxième hypothèse pour expliquer la forte volubilité de ce professeur, est de l'attribuer à une plus grande attention portée aux difficultés de compréhension en relation avec leur maîtrise faible de la langue, ce qui a été confirmé par l'enseignant lors de l'entretien post. Enfin, la troisième suggère une routine construite par le professeur en raison du contexte ZEP. II est indéniable que le « milieu difficile » qui caractérise l'établissement routinise des formes d'intervention visant à rappeler sans cesse à l'ordre les élèves pour prévenir tout comportements déviants (Van Zanten, 2001).

\section{Conclusion}

Cette étude exploratoire sur l'enseignement en ZEP suggère que de nombreuses contraintes pèsent sur les pratiques d'intervention. L'éclatement du savoir sous formes de micro tâches décontextualisées, les efforts de contrôle des élèves et de leur action motrice, le grand nombre des interactions verbales permettent de repérer des stratégies d'enrôlement des élèves dans les espaces de travail et dans le tempo de la séance. Ces stratégies concourent indéniablement au maintien de la relation didactique et expliquent qu'aucun comportement déviant, au sens de Toussignant (1985), n'ait été observé. En fait, comme les élèves sont tout le temps interpellés, nous ne constatons pas d'indiscipline. Par contre, l'évolution des interactions a pu mettre en évidence des comportements de transformation de tâches et les principaux résultats de l'étude confirment une certaine forme de "réduction des enjeux des savoirs » au fil des interactions. Mais, ils suggèrent, aussi, une ingéniosité didactique au sens développé par Amade-Escot (2001), qui est susceptible d'avoir des effets positifs sur les apprentissages dès lors que les milieux didactiques proposés aux élèves laissent certaines marges de manœuvre aux élèves. Ces propos recoupent en partie, pour l'enseignant observé en ZEP, les résultats de Badreau et Dhellemmes (1998) qui ont montré qu'il est possible de maintenir un enseignement centré sur les 
acquisitions. Ils plaident pour un développement des études didactiques sur l'enseignement en " milieu difficile » afin de mieux connaître les assujettissements auxquels sont confrontés élèves et enseignants.

\section{Bibliographie}

Amade-Escot, C. (2001). De l'usage des théories de l'enseignant : questions soulevées par l'étude des contrats didactiques en éducation physique. In A. Mercier, G. Lemoyne et A. Rouchier (Eds.) Le Génie Didactique. Usages et mésusages des théories de l'enseignement (pp. 23-41). Bruxelles : De Boëck, Perspectives en Education et Formation.

Amade-Escot, C. (2003). Didactique de l'éducation physique : état des recherches. Paris : Editions Revues EPS.

Badreau, J. et Dhellemmes, R. (1998). Enseigner l'éducation physique à des élèves en difficultés et / ou difficile. Recherche dite " édifice ». Paris et Nord Pas de Calais, IUFM.

Bautier E. et Rochex, J.Y. (1997). Apprendre : des malentendus qui font la différence. In J.P. Terrail (Dir). La scolarisation de la France : Critique de l'état des lieux (pp.105-122). Paris : La dispute.

Butlen, D., Barbier-Peltier, M-L. et Pézart, M. (2002). Nommés en REP, comment font-ils ? Pratiques de professeurs d'écoles enseignant les mathématiques en REP. Revue Française de pédagogie, 140, 41-52.

Mercier, A et Salin, M.H. (1988). L'analyse a priori, outil pour l'observation. In Actes de l'Université d'été de didactique des mathématiques. (pp. 203-207).

Miles, M.B. et Huberman, M. (1991). Analyse des données qualitatives : recueil de nouvelles méthodes. Bruxelles : De Boëck.

Peltier-Barbier, M-L. (2004). Dur d'enseigner en ZEP. Analyse des pratiques des professeurs enseignant en ZEP. Grenoble : La Pensée Sauvage.

Poggi-Combaz, M.P. (2002). Distribution des contenus d'enseignements en EPS au collège selon les caractéristiques sociales du public scolaire : des différences non aléatoires. Revue Française de Pédagogie, 139, 53-69.

Schubauer-Léoni, M.L. et Leutenegger, F. (2002). Expliquer et comprendre dans une approche clinique/expérimentale du didactique ordinaire. In F. Leutenegger 
et M. Saada-Robert (Eds). Expliquer et comprendre en sciences de l'éducation. (pp. 227-251). Bruxelles : De Boëck, Raisons éducatives.

Toussignant, M. (1985). Le degré de coopération des étudiants: une source d'hypothèses d'action pour l'enseignant. La Revue Québécoise de l'activité physique, 3, 69-74.

Van Zanten, A. (2001). La construction des normes professionnelles. In L'école de la périphérie. Scolarité et ségrégation en banlieue. (pp. 209-264). Paris, PUF. 